\title{
Baclofen to prevent agitation in alcohol- addicted patients in the ICU: study protocol for a randomised controlled trial
}

Mickael Vourc'h ${ }^{1}$, Fanny Feuillet ${ }^{2,3}$, Pierre-Joachim Mahe ${ }^{1}$, Véronique Sebille ${ }^{2,3}$, Karim Asehnoune $^{1 *}$ and The BACLOREA trial group

\begin{abstract}
Background: Alcohol is the leading psychoactive substance consumed in France, with about 15 million regular consumers. The National institute on Alcohol Abuse and Alcoholism (NIAAA) considers alcohol abuse to be more than 14 units of alcohol a week for men and 7 units for women. The specific complication of alcoholism is the alcohol withdrawal syndrome. Its incidence reaches up to $30 \%$ and its main complications are delirium tremens, restlessness, extended hospital stay, higher morbidity, and psychiatric and cognitive impairment. Without appropriate treatment, delirium tremens can lead to death in up to $50 \%$ of patients.
\end{abstract}

Methods/design: This prospective, double-blind, randomised controlled study versus placebo will be conducted in twelve French intensive care units (ICU). Patients with an alcohol intake level higher than the NIAAA threshold, who are under mechanical ventilation, will be included. The primary objective is to determine whether baclofen is more efficient than placebo in preventing restlessness-related side effects in the ICU. Secondary outcomes include mechanical ventilation duration, length of ICU stay, and cumulative doses of sedatives and painkillers received within 28 days of ICU admission. Restlessness-related side effects in the ICU are defined as unplanned extubation, medical disposal removal (such as urinary catheter, venous or arterial line or surgical drain), falling out of bed, ICU runaway (leaving ICU without physician's approval), immobilisation device removal, self-aggression or aggression towards medical staff. Daily doses of baclofen/placebo will be guided by daily creatinine clearance assessment.

Discussion: Restlessness in alcoholic patients is a life-threatening issue in ICUs. BACLOREA is a randomised study assessing the capacity of baclofen to prevent agitation in mechanically ventilated patients. Enrolment of 314 patients will begin in June 2016 and is expected to end in October 2018.

Trial registration: ClinicalTrials.gov Identifier: NCT02723383, registered on 3 March 2016.

Keywords: Alcoholism, Intensive care, Baclofen, Restlessness, Delirium tremens, Alcohol withdrawal syndrome Abbreviations: ANSM, ANSM French medicinal and health products safety agency; CIWA-Ar, Clinical Institute Withdrawal Assessment of Alcohol Scale, revised; Cl, Clearance; CRA, Clinical research associate; CT, Computed tomodensitometry; CWHD, Continuous veno-venous haemodialysis; DSMB, Data and Safety Monitoring Board; DTs, Delirium tremens; eCRF, Electronic case report form; ESEMED, European Study of the Epidemiology of Mental Disorders; IHD, Intermittent haemodialysis; ICU, Intensive care unit; IGSII, Simplified Gravity Index; MDRD, modification of diet in renal disease; NIAAA, National Institute on Alcohol Abuse and Alcoholism; SAS, Riker Sedation Agitation Scale; SOFA, Sequential Organ Failure Assessment; VFD, Ventilation-free days

\footnotetext{
* Correspondence: Karim.asehnoune@chu-nantes.fr

${ }^{1}$ Departments of Anaesthesiology and Surgical Intensive Care, Hôtel-Dieu,

University Hospital of Nantes, 44093 Nantes, France

Full list of author information is available at the end of the article
} 


\section{Background}

Alcohol is the leading psychoactive substance consumed in France with about 15 million regular consumers. The ESEMED4 study in 2001 [1] registered psychiatric disorders in about $10 \%$ of men and $2.5 \%$ of women with abusive alcohol intake. In 2013, alcohol was responsible for one death every $12 \mathrm{~min}$ in France [2]. In general hospitals, 20 to $35 \%$ of men and $10 \%$ of women suffer from chronic alcoholism [3]. In an intensive care unit (ICU), $28 \%$ of admissions are related to abusive alcohol intake [4]. The National institute on Alcohol Abuse and Alcoholism (NIAAA) considers alcohol abuse to be more than 14 units of alcohol a week for men and 7 units for women.

ICU admission is an independent risk factor for delirium [5-7], pain-related health care issues, sleeping problems and neurological dysfunction [8-10]. The specific complication of alcoholism is alcohol withdrawal syndrome. Its incidence reaches up to $30 \%$ [11] and its main complications are delirium tremens (DTs), restlessness, extended hospital stay, higher morbidity, and psychiatric and cognitive impairment [12-14]. Without appropriate treatment, DTs can lead to death in $50 \%$ of patients [15]. Alcohol abuse leads to specific neurological dysfunction characterised by a fall in neuronal density [16] and consequently a higher risk of convulsion, confusion and restlessness [17]. Moreover, alcoholism also worsens vital prognosis $[18,19]$ and increases the morbidity and mortality of surgical patients $[11,20]$.

Alcohol withdrawal syndrome and delirium are commonly encountered in ICUs but standard treatment fails to reduce restlessness and related complications. Agitation related to DTs can lead to unplanned extubation and medical disposal removal and requires deep sedation, increasing the time on the ventilator. The beneficial effects of baclofen in preventing such a condition have never been reported in ICUs for at-risk drinkers.

Baclofen has been commercialised since 1974 and its target is the type-B $\gamma$-aminobutyric acid (GABA) receptor. In at-risk patients, this drug can reduce alcohol craving. Randomised studies have reported its efficiency in reducing both alcohol withdrawal syndrome [21, 22] and alcohol intake $[23,24]$ with conclusive safety data. Doses of up to $400 \mathrm{mg}$ a day are commonly used, with very few side effects, in outpatients with working lives. Sixty to seventy percent of baclofen-treated patients reached total abstinence with a mean daily dose of $147 \mathrm{mg}$ [25]. These data, taken altogether, have led the French medicinal and health products safety agency (ANSM) to advocate new randomised controlled trials to assess both the safety and efficiency of high doses.

Our objective is to take advantage of sedation and mechanical ventilation to allow clinicians to deliver high doses of baclofen without neuropsychiatric side effects for quick and marked effects, in an attempt to control restlessness, craving and related morbidity in ICU.

\section{Methods/design \\ Design}

BACLOREA is a prospective, multicentre, double-blind, randomised controlled study versus placebo, performed in at-risk drinkers according to NIAAA criteria. We will assess the superiority of baclofen compared to placebo in preventing restlessness-related complications. The randomisation sequence will be computer-generated and stratified at the 11 participating centres. This study follows the international recommendations for interventional trials (see the SPIRIT checklist in Additional file 1).

\section{Hypothesis}

Early administration of baclofen to at-risk drinkers may reduce restlessness and its related complications when sedation is interrupted. We hypothesise that the administration of baclofen can reduce the incidence of patients who have at least one complication of agitation, including: unplanned extubation, medical disposal removal, falling out of bed, ICU runaway, immobilisation device removal, and self-aggression or aggression toward medical staff from 42 to $27 \%$.

\section{Trial intervention}

After randomisation, the patients will receive doubleblind baclofen or placebo. Daily doses will be adapted to daily modification of the diet in renal disease (MDRD) creatinine clearance from 150 to $50 \mathrm{mg}$ (see Table 1). On the day of randomisation, the patient will receive the full daily dose in a one-shot administration. Then daily doses will be divided into three intakes on the following days. During the mechanical ventilation period, the treatment/placebo will be administered via the nasogastric feeding tube. After extubation, the treatment will be administered either via the nasogastric tube or the oral route. The doses were determined after a preliminary pilot study and previous trial analysis regarding efficiency and safety data [25].

The treatment will be tapered over a 7-day period after extubation or tracheotomy, or after the 15th day of treatment if the patient is still under mechanical ventilation. Treatment will be interrupted as described in Table 2.

Clinical and biological safety data will be collected throughout the treatment period and the following 10 days, including blood count and liver function tests (see Additional file 2: SPIRIT Figure 3).

\section{Concomitant medication/treatment}

All other interventions will be performed at the discretion of the attending physicians. Participating ICUs will 
Table 1 Doses of baclofen or placebo adjusted on daily assessment of the patient's creatinine clearance

\begin{tabular}{lll}
\hline MDRD & D1 Loading dose & D2 and following days until extubation/tracheotomy at D15 \\
\hline $\mathrm{Cl} \geq 90$ & $150 \mathrm{mg}$ & $50-50-50$ \\
$\mathrm{Cl} 89-60$ & $100 \mathrm{mg}$ & $30-20-50$ \\
$\mathrm{Cl} 30-59$ & $70 \mathrm{mg}$ & $20-20-30$ \\
$\mathrm{Cl}<30$ or continuous HD & $50 \mathrm{mg}$ & $20-10-20$ \\
$\mathrm{HD}$ & $50 \mathrm{mg}$ & $50 \mathrm{mg}$ before each session \\
$\mathrm{Cl}<15$ without $\mathrm{HD} / \mathrm{HD}$ & $50 \mathrm{mg}$ & No administration \\
\hline
\end{tabular}

$C l$ renal clearance, $D$ day, $H D$ haemodialysis, $I H D$ intermittent haemodialysis, MDRD modification of the diet in renal disease

monitor sedation and pain in ventilated patients using specific scales according to international guidelines [26].

\section{Population}

At ICU admission, patients with a mechanical ventilation (MV) duration expected to be equal to or longer than 24 h may be included.

For patients with tracheal intubation at ICU admission:

- If intubation was performed less than $48 \mathrm{~h}$ before admission, inclusion can be performed within $24 \mathrm{~h}$ after admission

- If intubation was performed $48 \mathrm{~h}$ or more before admission, inclusion cannot be performed

For patients without tracheal intubation at ICU admission:

- If intubation was performed within the first $48 \mathrm{~h}$ after admission, inclusion can be performed in the first $24 \mathrm{~h}$ after intubation

- If intubation was performed at or after $48 \mathrm{~h}$, inclusion cannot be performed
All patients, whatever the severity of organ failure, would be eligible for screening inclusion.

\section{Inclusion criteria}

Adults aged from 18 to 70 years under mechanical ventilation for 1 day or more with an estimated alcohol intake of:

- 14 units of alcohol per week during the month before hospitalisation for men aged 18 to 64

- 7 units of alcohol per week during the month before hospitalisation for women or men older than 65

\section{Exclusion criteria}

Patients fulfilling one or more of the following criteria will not be included:

- Baclofen administration before ICU admission (personal treatment or single administration)

- Pregnancy

- Porphyria

- Burn injuries on ICU admission

- Personal treatment including gamma-hydroxybutyric acid (Alcover/Xyrem)

Table 2 Gradual interruption of baclofen or placebo after extubation or tracheotomy or on the 15th day of treatment

\begin{tabular}{|c|c|c|c|c|c|}
\hline $\begin{array}{l}\text { Dose administered on the day } \\
\text { of extubation/tracheotomy/15th day }\end{array}$ & $150 \mathrm{mg}$ & $100 \mathrm{mg}$ & 70 mg & $50 \mathrm{mg}$ & IHD 50 mg \\
\hline Day +1 & 100 mg (20-30-50) & 70 mg (20-20-30) & $50 \mathrm{mg}(10-20-20)$ & 30 mg (10-10-10) & $\begin{array}{l}30 \mathrm{mg} \text { before dialysis } \\
\text { (no administration if the } \\
\text { patient is not dialysed) }\end{array}$ \\
\hline Day +2 & 70 mg (20-20-30) & 50 mg (10-20-20) & 30 mg (10-10-10) & 20 mg (10-0-10) & $\begin{array}{l}30 \mathrm{mg} \text { before dialysis } \\
\text { (no administration if the } \\
\text { patient is not dialysed) }\end{array}$ \\
\hline Day +3 & 50 mg (10-20-20) & 30 mg (10-10-10) & 20 mg (10-0-10) & 10 mg (10-0-0) & $\begin{array}{l}10 \mathrm{mg} \text { before dialysis } \\
\text { (no administration if the } \\
\text { patient is not dialysed) }\end{array}$ \\
\hline Day +4 & 30 mg (10-10-10) & 20 mg (10-0-10) & 10 mg (10-0-0) & Stop & Stop \\
\hline Day +5 & 20 mg (10-0-10) & 10 mg (10-0-0) & Stop & & \\
\hline Day +6 & 10 mg (10-0-) & Stop & & & \\
\hline Day +7 & Stop & & & & \\
\hline
\end{tabular}


- Recent stroke or subarachnoid haemorrhage or head trauma with radiological evidence

- Paraplegia or tetraplegia

- Cardiac arrest with resuscitation manoeuvres before or after ICU admission

- Contraindication to enteral drug administration

- Lack of social protection

- Hypersensitivity to baclofen

- Coeliac disease

- Refractory epilepsy

- Dementia, schizophrenia, bipolar disorder or severe depression

- Parkinson's disease

- Health care limitation owing to a poor prognosis

- Tracheotomy on ICU admission

\section{Randomisation and blinding}

The clinicians will perform screening, enrolment and randomisation. Randomisation is centralised, web-based and accessible $24 \mathrm{~h}$ a day according to the allocation list on https://www.dirc-hugo-online.org/csonline/. Randomisation will be stratified at the centres using a fixed block size of 1:1 and a randomisation number will be allocated to each patient. Baclofen and placebo will be prepared and 'blinded' at Nantes University Hospital pharmacy. The independent trial statistician, patients, clinicians and nurses will be blinded for the allocation of treatment.

\section{Primary outcome measure}

Composite score: occurrence (yes or no) of agitationrelated adverse events during treatment/placebo administration with at least one sign out of the following:

- Unplanned extubation

- Medical disposal removal

- Falling out of bed

- ICU runaway

- Immobilisation device removal

- Self-aggression or aggression towards medical staff

\section{Secondary outcome measure}

- Composite score: occurrence (yes or no) of agitation-related adverse events during treatment/ placebo administration or 28-day mortality

- Adverse event (yes or no) related to agitation within 28 days of ICU admission

- Number of adverse events related to agitation occurring during ICU hospitalisation within 28 days of ICU admission

- Restlessness requiring rapid intravenous or intramuscular administration of hypnotic or neuroleptic drugs within 28 days of ICU admission
- Extubation failure defined as reintubation within the next $48 \mathrm{~h}$

- Tracheotomy for failure of mechanical ventilation weaning

- Infections acquired in the ICU: urinary infection, pneumonia, catheter infection or bacteraemia

- Total doses of sedatives and painkillers received in the ICU within 28 days of ICU admission

- Riker Sedation Agitation Scale (SAS) in the ICU within 28 days of ICU admission

- Daily Clinical Institute Withdrawal Assessment of Alcohol Scale, revised (CIWA-Ar) alcohol withdrawal score during the week following extubation

- Duration of mechanical ventilation

- Ventilation-free days (VFD) at day 28

- Length of ICU stay

- Length of total hospitalisation

- Death in ICU, at days 28 and 90

- Death during hospital stay

\section{Participant withdrawal}

Patients will be excluded from the trial if they or their next-of-kin withdraw their consent. As a result, the investigator will immediately interrupt treatment administration.

If the patient does not object to information on the primary outcome measure being obtained, the data will be collected and analysed. The data will be fully erased if the patient refuses this permission.

\section{Protocol suspension and severe adverse reactions}

The treatment could be discontinued:

- Temporarily if:

Renal clearance falls below $15 \mathrm{ml} / \mathrm{min}$ without haemodialysis support

$\bigcirc$ Liver tests (amino-transferase enzymes) are more than 20 times the normal

$\bigcirc$ Heart rate falls below 50 BPM with haemodynamic consequences

- Definitively if:

Allergic symptoms appear

Heart rate falls below 35 BPM whatever the haemodynamic profile

$\bigcirc$ Non-reactive uni/bilateral mydriasis develops

$\bigcirc$ Patient has a seizure in the ICU

Patient has a stroke in the ICU with computed tomodensitometry $(\mathrm{CT})$ scan confirmation

$\bigcirc$ Eyes not open $72 \mathrm{~h}$ after total sedation is interrupted

\section{Statistics}

We hypothesised that baclofen administration can reduce the incidence of patients with at least one 
complication of agitation, using a two-sided test with an $\alpha$ of $5 \%$ and $80 \%$ power, from 42 to $27 \%$. This hypothesis in the placebo group is based on personal data. As a result, 314 patients are needed. Our study will include patients whatever their severity. Some patients may die before sedation interruption thus altering the power of the study. We therefore plan an interim analysis (after $50 \%$ of inclusions), to adjust the sample size to maintain the statistical power. Friede and Kieser's method [27, 28] allows both the initial hypothesis and the type- 1 error planned a priori to be maintained. The analysis will be stratified at the participating centres. Statistical analysis will take place on an intention-to-treat principle. For the primary outcome, a logistic regression model will be applied to compare the proportion of agitation between the two treatment arms. Two sensitivity analyses will be performed and will consider dead patients to be without agitation, or as patients with or without agitation. A per-protocol analysis will also be performed to exclude patients with any of the exclusion criteria or without the inclusion criteria, or patients with incomplete treatment administration.

For the secondary outcomes, a Poisson regression model will be applied to compare the number of adverse events related to agitation, between the two treatment arms. Both length of ICU stay and mechanical ventilation will be compared with a log-rank test. Logistic regression will be used to compare binary qualitative secondary endpoints.

Normally distributed variables will be expressed as mean and standard deviation; nonnormally distributed variables will be expressed as median and interquartile range. Categorical variables will be expressed as numbers and percentages.

\section{Track record}

Data will be recorded in the web-based electronic case report form (eCRF) by trial site staff. Characteristics at baseline will be gathered: age, site of inclusion, sex, weight, height, medical history, reason for ICU admission, alcohol consumption, kidney function, Simplified Gravity Index (IGSII) and Sequential Organ Failure Assessment (SOFA) scores on admission. During ICU hospitalisation we will assess: cumulative doses of all sedative drugs, agitation and alcohol withdrawal scores, agitation-related adverse events, blood tests results regarding alcohol test on admission, liver function tests and blood count during treatment, acquired infection, length of ICU stay, mechanical ventilation and whole hospitalisation. To preserve confidentiality of participant's personal information, data will be key-coded using alphanumerical numbers. To minimise missing data, primary and secondary outcome will be notified daily. Patients will be monitored until day 90 and a supplemental telephonic contact will promote complete follow-up.

\section{Monitoring}

Monitoring will follow Good Clinical Practice principles and will be performed by the independent promotion department of Nantes University Hospital Research Management Unit. A clinical research associate (CRA) will visit each centre every 6 months to control the quality of the recorded data. The CRA will be able to check the case report forms and medical records. The following data will be assessed:

- Written informed consent

- Flow chart filled in for included and excluded patients

- Trial progress

- Primary and secondary outcome collection

- Treatment-related severe adverse events

- Stock management of the study treatment

Participating centres agree to accept the promoter's quality audits.

\section{Trial status}

Patients from 11 ICUs are expected to be included over a 2-year inclusion period. We plan to include two patients per ICU per month.

2015: protocol approval by the Ethics Committee and French national drug safety agency.

June 2016-2018: inclusions

Beginning of 2017: interim analysis

2018: closing of the database. We will submit our manuscript during the second half of 2018.

\section{Discussion}

Restlessness in alcoholic patients is a life-threatening issue in ICUs. Baclofen usefullness was already reported to treat alcohol withdrawal syndrome, that's why its accuracy in ICU had to be assessed by large multicentre studies. Invasive Mechanical ventilation and continuous tight monitoring will allowed to introduce relatively high doses of Baclofen to reach fast effective plasma drug concentration and ensure patient security. In parallel, reducing agitation may avoid other hypnotic drugs administration and so reduce their side effects: Mechanical ventilation duration and its complications, delirium and neuro-psychiatric aftereffects.

\section{Additional files}

Additional file 1: SPIRIT checklist. (DOC $117 \mathrm{~kb}$ )

Additional file 2: Figure 3 Study schedule. Caption of Figure 3: ICU, Intensive care unit; SAS, Riker Sedation Agitation Scale; CIWA-Ar, Clinical Institute Withdrawal Assessment of Alcohol Scale, revised; NIAAA, National Institute on Alcohol Abuse and Alcoholism. (DOCX 136 kb) 


\section{Acknowledgements}

This study is an investigator-initiated trial, funded by the French Ministry of Health (Interregion French Clinical Hospital Research Programme grant (PHRCI 2014-API14/N/096).

BACLOREA trial group. Alphabetical order of centres:

Surgical Intensive Care Unit, University Hospital, (4 rue Larrey), Angers,

(44933), France

Sigismond Lasocki: silasocki@chu-angers.fr

Soizic Gergaud: sogergaud@chu-angers.fr

Thomas Gaillard: togaillard@chu-angers.fr

Intensive Care Unit, Hospital, (Les Oudairies), La Roche-sur-Yon, (85925),

France

Jean-Baptiste Lascarrou: jean-baptiste.lascarrou@chd-vendee.fr

Isabelle Vinatier: isabelle.vinatier@chd-vendee.fr

Laurent Martin-Lefevre: Laurent.martin-lefevre@chd-vendee.fr

Aihem Yehia: aihem.yehia@chd-vendee.fr

Gwenhael Colin: gwenhael.colin@chd-vendee.fr

Christine Lebert: Christine.lebert@chd-vendee.fr

Maud Fiancette: maud.fiancette@chd-vendee.fr

Matthieu Henry-Lagarrigue: matthieu.henry-lagarrigue@chd-vendee.fr

Jean-Claude Lacherade: jean-claude.lacherade@chd-vendee.fr

Surgical Intensive Care Unit, University Hospital, (80 avenue Augustin Fliche),

Montpellier, (34090), France

Samir Jaber: s-jaber@chu-montpellier.fr

Audrey De Jong: a-de_jong@chu-montpellier.fr

Gerald Chanques: g-chanques@chu-montpellier.fr

Surgical Intensive Care Unit, University Hospital, (1 place Alexis Ricordeau), Nantes (44093), France.

Karim Asehnoune: karim.asehnoune@chu-nantes.fr

Mickael Vourc'h: mickael.vourch@chu-nantes.fr

Pierre-Joachim Mahe: pierrejoachim.mahe@chu-nantes.fr

Antoine Roquilly: antoine.roquilly@chu-nantes.fr

Raphaël Cinotti: raphael.cinotti@chu-nantes.fr

Dominique Demeure Dit Latte: dominique.demeureditlatte@chu-nantes.fr

Medical Intensive Care Unit, University Hospital, (1 place Alexis Ricordeau),

Nantes (44093), France.

Jean Reignier: jean.reignier@chu-nantes.fr

Charlotte Garret: charlotte.garret@chu-nantes.fr

Cédric Bretonniere: cedric.bretonniere@chu-nantes.fr

Noëlle Brule: noelle.brule@chu-nantes.fr

Laurent Nicolet: laurent.nicolet@chu-nantes.fr

Olivier Zambon: olivier.zambon@chu-nantes.fr

Surgical Intensive Care Unit, University Hospital, (2 rue de la Miletrie), Poitiers, (86021), France

Olivier Mimoz: olivier.mimoz@chu-poitiers.fr

Intensive Care Unit, Hospital, (14 avenue Yves Thépot), Quimper, (29107), France

Mikael Moriconi: m.moriconi@ch-cornouaille.fr

Medical Intensive Care Unit, University Hospital, (rue Henri Le Guilloux),

Rennes, (35043), France

Arnaud Gacoin: arnaud.gacouin@chu-rennes.fr

Jean-Marc Tadie: jeanmarc.tadie@chu-rennes.fr

Yves Le Tulzo: yves.le.tulzo@chu-rennes.fr

Surgical Intensive Care Unit, University Hospital, (184 rue du Faubourg Saint-

Antoine), Saint-Antoine, (75571), France

Thomas Lescot: thomas.lescot@sat.aphp.fr

Intensive Care Unit, Hospital, (11 blvd. Charpak), Saint-Nazaire (44606), France

Paul Morin: p.morin@ch-saintnazaire.fr

Maud Jonas: m.jonas@ch-saintnazaire.fr

Freddy Thibaut: f.thibaut@ch-saintnazaire.fr

Surgical Intensive Care Unit, University Hospital, (avenue de la République),

Tours, (37170), France

Martine Ferrandiere: m.ferrandiere@chu-tours.fr

\section{Funding}

The BACLOREA study is supported by the French Ministry of Health (Interregion French Clinical Hospital Research Programme grant (PHRCl 2014-API14/N/096). Nantes University Hospital sponsors the study.

Availability of data and materials

All data will be available.

\section{Authors' contributions}

KA obtained fundings. KA, PJM and MV designed the study. VS and FF planned the statistical analysis. KA and MV will have full access to the final trial dataset. All authors read and approved the final manuscript.

\section{Competing interests}

The authors declare that they have no competing interests.

\section{Ethics approval and consent to participate}

Patient screening will start after Ethics Committee and French regulation agency approvals have been obtained. State of the art suggests the potential benefits of baclofen administration to patients suffering from alcoholism without relevant or life-threatening side effects. Furthermore, if baclofen succeeds in preventing restlessness in the ICU, its use may become widely generalised since agitation is a serious issue in ICUs. The Ethics Committee ('Comité de Protection des Personnes ouest II' for Angers, France) approved this study protocol in 2015 ( $N^{\circ}$ CPP 2015/09). The French National Agency for Medicines and Health Products Safety gave its approval on 3 March 2016. Most of the patients assessed for enrolment will not be able to provide written consent and so will be included after emergency or next-of-kin written consent has been obtained. Clinicians will ensure information and consent collection. Baclofen must be delivered early after admission in order to reach an efficient plasma concentration as quickly as possible. Very few patients will be enrolled after obtaining their self-written consent. As soon as patients recover consciousness and have a compatible mental status, clinicians will ask them for their informed written consent.

\section{Publication plan}

The trial is registered on www.Clinicaltrials.gov. Upon trial completion the main manuscript will be submitted for publication.

\section{Safety}

After the clinicians' electronic report, the Pharmacovigilance Unit of Nantes University Hospital will assess and manage solicited or spontaneously reported adverse events.

A Data and Safety Monitoring Board (DSMB) will be available for this study, and will include a methodologist, an intensivist and a specialist in alcohol abuse. Members must be independent and not employed by the study sponsor. The Monitoring Committee is responsible for the following:

- Providing independent medical expertise when necessary

- Providing independent expertise concerning the occurrence of complications

- Judging whether the trial needs to be stopped on grounds of safety or efficacy after interim analysis

The Monitoring Committee can request the promoter to unblind treatment allocation in case of a serious adverse event.

Baclofen plasma concentration will be blindly assessed in three centres and analyses will be centralised by the pharmacology laboratory in Nantes University Hospital. Results will be anonymously analysed by the DSMB.

\section{Author details}

${ }^{1}$ Departments of Anaesthesiology and Surgical Intensive Care, Hôtel-Dieu, University Hospital of Nantes, 44093 Nantes, France. ${ }^{2}$ Biometry Platform, Research Promotion Department, University Hospital of Nantes, 44093 Nantes, France. ${ }^{3}$ EA 4275 SPHERE Methods for Patient-centered Outcomes and Health Research, Nantes University, 44035 Nantes, France.

Received: 19 April 2016 Accepted: 4 August 2016

Published online: 19 August 2016

\section{References}

1. Alonso J, Ferrer M, Romera B. The European Study of the Epidemiology of Mental Disorders (ESEMeD/MHEDEA 2000) project: rationale and methods. 2002;11(2):55-67. doi:10.1002/mpr.123.

2. Guerin S, Laplanche A, Dunant A. Alcohol-attributable mortality in France. 2013;23(4):588-93. doi:10.1093/eurpub/ckt015.

3. Vourc'h G, Hatton F, Tiret L. Epidemiologic study of anesthesia complications in France. Bull Acad Natl Med. 1983;167(8):939-45. 
4. Canarelli T, Cadet A, Palle C. Indicateurs de la morbidité et de la mortalité liées à l'alcool en France. Bull Epidemiol Hebdo. 2006;n³4-35:252-5.

5. Sanders RD, Pandharipande PP, Davidson AJ, Ma D, Maze M. Anticipating and managing postoperative delirium and cognitive decline in adults. BMJ. 2011;343:d4331.

6. Pandharipande P, Shintani A, Peterson J. Lorazepam is an independent risk factor for transitioning to delirium in intensive care unit patients. Anesthesiology. 2006;104(1):21-6.

7. Pisani MA, Murphy TE, Araujo KLB, Slattum P, Van Ness PH, Inouye SK. Benzodiazepine and opioid use and the duration of intensive care unit delirium in an older population. Crit Care Med. 2009;37(1):177-83. doi:10. 1097/CCM.0b013e318192fcf9.

8. Lavie P. Sleep disturbances in the wake of traumatic events. N Engl J Med. 2001;345(25):1825-32. doi:10.1056/NEJMra012893.

9. Liang C, Chen J, Gu W. Chronic alcoholism increases the induction dose of propofol. Acta Anaesthesiol Scand. 2011;55(9):1113-7. doi:10.1111/j.1399-6576. 2011.02497.x.

10. Wolfson B, Freed B. Influence of alcohol on anesthetic requirements and acute toxicity. Anesthesia Analg. 1980;59(11):826-30.

11. Spies CD, Nordmann A, Brummer $G$, et al. Intensive care unit stay is prolonged in chronic alcoholic men following tumor resection of the upper digestive tract. Acta Anaesthesiol Scand. 1996;40(6):649-56.

12. Levkoff SE, Evans DA, Liptzin B, et al. Delirium. The occurrence and persistence of symptoms among elderly hospitalized patients. Arch Intern Med. 1992;152(2):334-40.

13. Francis J, Martin D, Kapoor WN. A prospective study of delirium in hospitalized elderly. JAMA. 1990;263(8):1097-101.

14. Ely EW, Shintani A, Truman B, et al. Delirium as a predictor of mortality in mechanically ventilated patients in the intensive care unit. JAMA 2004;291(14):1753-62. doi:10.1001/jama.291.14.1753.

15. Griffin RE, Gross GA, Teitelbaum HS. Delirium tremens: a review. J Am Osteopath Assoc. 1993;93(9):924-929-32-935.

16. Kril JJ, Halliday GM. Brain shrinkage in alcoholics: a decade on and what have we learned? Prog Neurobiol. 1999;58(4):381-7.

17. Neiman J. Alcohol as a risk factor for brain damage: neurologic aspects. Alcohol Clin Exp Res. 1998;22(7 Suppl):346S-51.

18. Gacouin A, Tadie JM, Sauvadet E. At-risk drinking is independently associated with ICU and one-year mortality in critically ill nontrauma patients*. Crit Care Med. 2014;42(4):860-7. doi:10.1097/CCM. 0000000000000041.

19. Clark BJ, Williams A, Feemster LMC, et al. Alcohol screening scores and 90-day outcomes in patients with acute lung injury. Crit Care Med. 2013;41(6):1518-25. doi:10.1097/CCM.0b013e318287f1bb.

20. Tønnesen H, Petersen KR, Højgaard L, et al. Postoperative morbidity among symptom-free alcohol misusers. Lancet. 1992;340(8815):334-7.

21. Addolorato $G$, Leggio $L$, Abenavoli $L$, et al. Baclofen in the treatment of alcohol withdrawal syndrome: a comparative study vs diazepam. Am J Med. 2006:119(3):276.e13-e18. doi:10.1016/j.amjmed.2005.08.042.

22. Lyon JE, Khan RA, Gessert CE, Larson PM, Renier CM. Treating alcohol withdrawal with oral baclofen: a randomized, double-blind, placebo-controlled trial. J Hosp Med. 2011;6(8):469-74. doi:10.1002/jhm.928.

23. Addolorato G, Leggio L, Ferrulli A, Cardone S. Effectiveness and safety of baclofen for maintenance of alcohol abstinence in alcohol-dependent patients with liver cirrhosis: randomised, double-blind controlled study. The Lancet. 2007:370(9603):1915-1922. doi: 10.1016/S0140-6736(07)61814-5.

24. Addolorato G, Leggio L, Ferrulli A, Cardone S, Bedogni G, Caputo F, et al. Dose-response effect of baclofen in reducing daily alcohol intake in alcohol dependence: secondary analysis of a randomized, double-blind, placebocontrolled trial. Alcohol and Alcoholism. 2011;46(3):312-317. doi:10.1093/ alcalc/agr017.

25. Rigal L, Alexandre-Dubroeucq C, de Beaurepaire R. Abstinence and 'low-risk' consumption 1 year after the initiation of high-dose baclofen: a retrospective study among 'high-risk' drinkers. Alcohol Alcohol. 2012;47(4): 439-42. doi:10.1093/alcalc/ags028.

26. Riker RR, Fraser GL. The new practice guidelines for pain, agitation, and delirium. Am J Crit Care. 2013;22(2):153-7. doi:10.4037/ajcc2013480.

27. Friede T, Kieser M. Sample size recalculation for binary data in internal pilot study designs. Pharm Stat. 2004;3(4):269-79. doi:10.1002/pst.140.

28. Friede T, Kieser M. Sample size recalculation in internal pilot study designs: a review. Biom J. 2006:48(4):537-55.

\section{Submit your next manuscript to BioMed Central and we will help you at every step:}

- We accept pre-submission inquiries

- Our selector tool helps you to find the most relevant journal

- We provide round the clock customer support

- Convenient online submission

- Thorough peer review

- Inclusion in PubMed and all major indexing services

- Maximum visibility for your research

Submit your manuscript at www.biomedcentral.com/submit
Biomed Central 